\title{
Sensitivity Analysis of a Crawl Gait Multi-objective Optimization System
}

\author{
Miguel Oliveira \\ Industrial Electronics \\ Department, School of \\ Engineering, University of \\ Minho \\ 4800-058 Guimaraes, Portugal \\ mcampos@dei.uminho.pt
}

\author{
Pedro Silva \\ Industrial Electronics \\ Department, School of \\ Engineering, University of \\ Minho \\ 4800-058 Guimaraes, Portugal \\ psilva@dei.uminho.pt \\ Lino Costa \\ Production Systems \\ Department, School of \\ Engineering,University of \\ Minho 4800-058 Guimaraes, \\ Portugal \\ lac@dps.uminho.pt
}

\author{
Cristina Santos \\ Industrial Electronics \\ Department, School of \\ Engineering, University of \\ Minho
}

4800-058 Guimaraes, Portugal

cristina@dei.uminho.pt

\begin{abstract}
This paper describes the analysis of a crawl gait multi-objective optimization system that combines bio-inspired Central Patterns Generators (CPGs) and a multi-objective evolutionary algorithm. In order to optimize the crawl gait, a multiobjective problem, an optimization system based on NSGAII allows to find a set of non-dominated solutions that correspond to different motor solutions

The experimental results highlight the effectiveness of this multi-objective approach.
\end{abstract}

\section{Categories and Subject Descriptors}

I.2.9 [Robotics]; G.1.6 [Optimization]:

\section{Keywords}

Evolutionary robotics, Multi-objective optimization, Genetic algorithms

\section{INTRODUCTION}

Legged robot locomotion is a complex problem that usually implies the control and coordination of multiple degrees of freedom. One possible bio-inspired solution is to apply nonlinear dynamical equations of high order with a multidimensional and irregular set of parameters, that model the biological nervous system.

The lack of knowledge about the dynamical behavior of the robot platform, the environment and the interaction

Permission to make digital or hard copies of all or part of this work for personal or classroom use is granted without fee provided that copies are not made or distributed for profit or commercial advantage and that copies bear this notice and the full citation on the first page. To copy otherwise, to republish, to post on servers or to redistribute to lists, requires prior specific permission and/or a fee.

GECCO'13, July 6-10, 2013, Amsterdam, The Netherlands.

Copyright 2013 ACM TBA ...\$10.00. among both; and the very large number of parameters makes the hand-tuning very hard to achieve and may not ensure the best results. So, biological inspired Evolutionary Computation (EC) appears as a possible choice for gait optimization of legged robots.

In [4] we have combined a proposed Central Pattern Generator (CPG) approach with a multi-objective optimization strategy, the elitist Nondominated Sorting Genetic Algorithm [2] (NSGA-II), that searches for the best set of CPG parameters that result in the desired crawl gait. Speed, stability and behavioral diversity were the evaluated criteria used to explore the parameter space of the network of CPGs.

In this work, we are particularly interested in studying the compromise solutions obtained to see if they reveal interesting features of the CPG parameters and in their relation to the addressed objectives. Thus, we proceed to a sensitivity analysis to assess the relationships between the parameters and the objectives and between the objectives themselves.

Results demonstrate the performance of the achieved robot walking.

\section{OPTIMIZATION SYSTEM}

The proposed network of CPGs generates trajectories for the robot hip pitch joints, and therefore to the robot limbs. The $x_{i}$ solutions of a modified Landau-Stuart oscillator, directly control each hip pitch joint of a limb, $i$, as follows

$$
\begin{aligned}
& \dot{x}_{i}=\alpha\left(\mu-r_{i}^{2}\right)\left(x_{i}-O_{i}\right)-\omega_{i} z_{i}, \\
& \dot{z}_{i}=\alpha\left(\mu-r_{i}^{2}\right) z_{i}+\omega_{i}\left(x_{i}-O_{i}\right),
\end{aligned}
$$

where $r_{i}=\sqrt{\left(x_{i}-O_{i}\right)^{2}+z_{i}^{2}}, \omega_{i}$ specifies the oscillation frequency (in $\mathrm{rads}^{-1}$ ) and $\mu$ value specifies the oscillation amplitude $A$, where $\mu=A^{2}$. This oscillator bifurcates from a stable fixed point at $\left(x_{i}, z_{i}\right)=\left(O_{i}, 0\right)$ (when $\left.\mu<0\right)$ to a structurally stable, harmonic limit cycle, for $\mu>0$, with offset $O_{i}$. Despite phase relationships, left and right legs have similar trajectories. This enables to minimize the decision vector $(\mathbf{x})$, see Table 1 . 
Table 1: CPG parameter bounds

\begin{tabular}{|c|c|c|c|c|c|c|c|}
\hline Parameter & $\mu_{\mathrm{FL}}$ & $O_{\mathrm{FL}}$ & $\mu_{\mathrm{HL}}$ & $O_{\mathrm{HL}}$ & $\omega_{\mathrm{sw}}(\mathrm{rad} / \mathrm{s})$ & $K_{\mathrm{FL}}\left({ }^{\circ}\right)$ & $K_{\mathrm{HL}}\left({ }^{\circ}\right)$ \\
\hline Lower bound (l) & 0.0001 & -1600 & 0.0001 & -400 & 1 & -30 & 5 \\
\hline Upper bound (u) & 3600 & 400 & 3600 & 1600 & 12 & 127 & 127 \\
\hline
\end{tabular}

Parameters are tuned according to the optimization system depicted in Figure 1. We use an optimization algorithm framework based on NSGA-II to search for the optimal set of the CPG parameters.

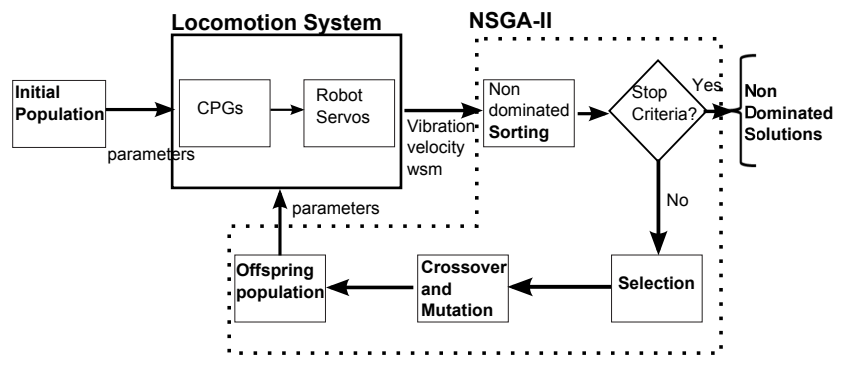

Figure 1: Optimization Locomotion System

We consider that the performance of a walking gait can be assessed in terms of the maximization of three objectives $(\mathbf{f}(\mathbf{x}))$, where $\mathbf{x}$ is a decision vector: the velocity (Vel), the wide stability margin (WSM) and the sensory motor behavioral diversity (BD) [3]. The constraints are handled according to method proposed by Deb [1].

\section{RESULTS}

Simulations were carried out in an ers-7 AIBO dog robot in Webots, a simulation software based on ODE, an open source physics engine for simulating 3D rigid body dynamics.In all experiments, we consider a population size of 100 chromosomes and a maximum number of generations of 200 . Each chromosome is evaluated during 12 seconds.

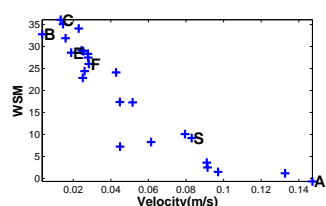

(a)

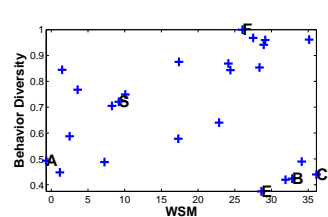

(c)

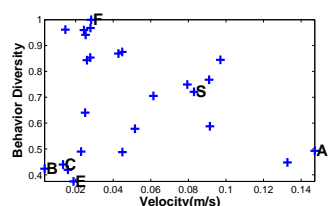

(b)

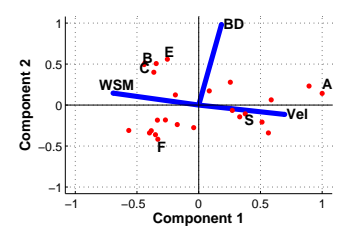

(d)
Figure 2: Non-dominated solutions in objective space (a,b,c figures) and PCA Biplot(d figure)

In Figure 2, the non-dominated solutions in terms of the objectives are presented. These graphs are $2 \mathrm{D}$ projections of the 3D objective space. We show the six extreme nondominated solutions (highest Vel A, lowest Vel B, highest WSM C, lowest WSM D, highest BD $\mathbf{E}$, and lowest BD F), as well as the ones of the hand-tuned solution and a non- dominated $\mathbf{S}$ solution which respects the midterm of all objectives.

The goal of the sensitivity analysis herein described is to study which and how the variation of the CPG parameters, influence the different objectives; to study the relationship between the different proposed objectives and to verify if any of the objectives is redundant and as such should be discarded.

The strongest significant Pearson correlation coefficient $(R)$ between the CPG parameters and objectives are: for Vel with $\mu_{\mathrm{FL}}(R=0.97), \omega_{\mathrm{sw}}(R=0.96)$ and $K_{\mathrm{HL}}(R=$ $-0.81)$; for WSM with $O_{\mathrm{FL}}(R=0.78), \omega_{\mathrm{sw}}(R=-0.79)$ and $K_{\mathrm{HL}}(R=-0.71)$; and for $\mathrm{BD}$ with $\mu_{\mathrm{FL}}(R=-0.83)$.

In order to verify the relationship between the objective functions, we calculate a Principal Component Analysis (PCA).

Note that component 1 and component2 explain approximately $99.5 \%$ of the variance. This enables us to use biplots (Fig $2 \mathrm{~d})$ ) in order to inspect the relations between objectives. The biplot shows that Vel and WSM have a negative correlation (in fact an angle of $\approx 180^{\circ}$ to both the other objectives) and $\mathrm{BD}$ is independent from the other objectives (angle $\approx 90^{\circ}$ to both the other objectives).

\section{CONCLUSIONS AND FUTURE WORKS}

In this article, we have addressed the sensitivity analysis of a previous locomotion optimization framework of a quadruped robot that walks with a slow walking gait. The proposed system combines a CPG based locomotion controller and NSGA-II.

The relationship between the objective functions analysis indicates that are no redundant objectives and that there is a strong negative correlation between the Vel and the WSM.

Currently, we are addressing the reality gap between the real and simulated robot problems.

\section{ACKNOWLEDGMENTS}

This work is funded by FEDER Funding supported by the Operational Program Competitive Factors - COMPETE and National Funding supported by the FCT - Portuguese Science Foundation through projects PEst-OE/EEI/LA0009 /2011 and project PTDC/EEACRO/ 100655/2008.

\section{REFERENCES}

[1] K. Deb. An efficient constraint handling method for genetic algorithms. In Computer Methods in Applied Mechanics and Engineering, pages 311-338, 1998.

[2] K. Deb, A. Pratap, S. Agarwal, and T. Meyarivan. A fast and elitist multiobjective genetic algorithm: Nsga-ii. Evolutionary Computation, IEEE Transactions on, 6(2):182 -197, Apr. 2002.

[3] S. Koos, J.-B. Mouret, and S. Doncieux. Crossing the reality gap in evolutionary robotics by promoting transferable controllers. In Proceedings of the 12th annual conference on Genetic and evolutionary computation, GECCO '10, pages 119-126, New York, NY, USA, 2010. ACM.

[4] M. Oliveira, C. P. Santos, L. Costa, and M. Ferreira. Multi-objective parameter cpg optimization for gait generation of a quadruped robot considering behavioral diversity. 2011 IEEE/RSJ International Conference on Intelligent Robots and Systems (IROS 2011), pages 2286-2291, 2011. 